Journal of Trauma and Acute Care Surgery, Publish Ahead of Print

DOI: $10.1097 /$ TA.0000000000002069

\title{
Blood Purification by Non-Selective Hemoadsorption Prevents Death after Traumatic \\ Brain Injury and Hemorrhagic Shock in Rats
}

Todd O. McKinley MD

Departments of Orthopaedic Surgery, Anatomy and Cell Biology; Indiana University School of Medicine.

Zhigang Lei $\mathrm{PhD}$

Department of Anatomy and Cell Biology; Indiana University School of Medicine;

zhigang.lei1@gmail.com

Yannik Kalbas MS

Universitätsspital Zürich; yannik.kalbas@gmail.com

Fletcher A. White $\mathrm{PhD}$

Department of Anesthesiology; Indiana University School of Medicine; fawhite@iu.edu

Zhongshang Shi MD

GHM Institute of CNS Regeneration; Jinan University; Guangzhou, China;

15625162467@163.com

\section{Fan $\mathrm{Wu} \mathrm{MD} \mathrm{PhD}$}

Department of Pediatrics; The Third Affiliated Hospital of Guangzhou Medical University;

Guangzhou, China; gdwufan@126.com

This is the author's manuscript of the article published in final edited form as:

McKinley, T. O., Lei, Z., Kalbas, Y., White, F. A., Shi, Z., Wu, F., ... Rodgers, R. B. (2018). Blood Purification by Non-Selective Hemoadsorption Prevents Death after Traumatic Brain Injury and Hemorrhagic Shock in Rats. Journal of Trauma and Acute Care Surgery, Publish Ahead of Print. https://doi.org/10.1097/TA.0000000000002069 
Zao C. Xu PhD

Department of Anatomy and Cell Biology; Indiana University School of Medicine; zcxu@iupui.edu

Richard B. Rodgers MD

Department of Neurosurgery; Indiana University School of Medicine; rrodgers@goodmancampbell.com

Corresponding author: Todd O. McKinley MD, Departments of Orthopaedic Surgery, Anatomy and Cell Biology; Indiana University School of Medicine. IU Health Methodist Hospital, Suite 535 MPC-1, Indianapolis, IN 46202, tmckinley@iuhealth.org, Office: 317-963-1966, Fax:

317-963-1955

Conflict of Interest: Material support was received from Cytosorbents (Monmouth Junction, NJ) for conducting the experiment.

Funding: This study was funded by the Indiana University Clinical and Translational Sciences Institute, Indianapolis, IN.

This work was presented at the 2017 Military Health System Research Symposium August $27^{\text {th }}$ $30^{\text {th }}, 2017$, Orlando, FL. 
Background: Patients who sustain traumatic brain injury (TBI) and concomitant hemorrhagic shock (HS) are at high risk of high-magnitude inflammation which can lead to poor outcomes and death. Blood purification by hemoadsorption (HA) offers an alternative intervention to reduce inflammation after injury. We tested the hypothesis that HA would reduce mortality in a rat model of TBI and HS.

Methods: Male Sprague Dawley rats were subjected to a combined injury of a controlled cortical impact (CCI) to their brain and pressure-controlled hemorrhagic shock (HS). Animals were subsequently instrumented with an extracorporeal blood circuit that passed through a cartridge for sham or experimental treatment. In experimental animals, the treatment cartridge was filled with proprietary beads (Cytosorbents; Monmouth Junction, NJ) that removed circulating molecules between $5 \mathrm{KDa}$ and $60 \mathrm{KDa}$. Sham rats had equivalent circulation but no blood purification. Serial blood samples were analyzed with multiplex technology to quantify changes in a trauma-relevant panel of immunologic mediators. The primary outcome was survival to $96 \mathrm{hr}$ post-injury.

Results: HA improved survival from $47 \%$ in sham treated rats to $86 \%$ in HA treated rats. There were no treatment-related changes in histologic appearance. HA affected biomarker concentrations both during the treatment and over the ensuing four days after injury. Distinct changes in biomarker concentrations were also measured in survivor and non-survivor rats from the entire cohort of rats indicating biomarker patterns associated with survival and death after injury.

Conclusions: Blood purification by non-selective HA is an effective intervention to prevent death in a combined TBI/HS rat model. HA changed circulating concentrations of multiple inmmunologically active mediators during the treatment time frame and after treatment. HA has been safely implemented in human patients with sepsis and may be a treatment option after injury.

Keywords: hemoadsorption; traumatic brain injury; hemorrhagic shock; cytokines; polytrauma 


\section{Background}

Patients sustaining traumatic brain injuries (TBI) are often multiply injured and frequently present with concomitant hemorrhagic shock (HS) ${ }^{1,2}$. In patients with HS, TBI increases the risk to develop high-magnitude sustained systemic inflammation, immunologic dysfunction, and multiple organ dysfunction syndrome (MODS) ${ }^{3-6}$. Conversely, the immunologic response and excessive inflammation resulting from HS exacerbate the severity of $\mathrm{TBI}^{7,8}$. Under physiologic conditions, the brain is largely isolated from systemic immune surveillance and inflammation by the blood brain barrier $(\mathrm{BBB})$. TBI disrupts this protective mechanism and systemic inflammation and the peripheral immune response to injury gain access to the brain while intracranial inflammation gains access to the systemic circulation ${ }^{6,9-11}$. The reciprocating effects of intracranial and systemic inflammatory responses propagate secondary injury to the brain ${ }^{8,12-}$ ${ }^{16}$ and peripheral organ dysfunction ${ }^{6,11}$.

Extensive research in animal models has demonstrated that pharmaceutical mitigation of intracranial inflammation improves histological scores and functional outcomes after TBI ${ }^{17}$. Accordingly, clinicians have attempted to improve outcomes after TBI by blocking inflammation to reduce secondary brain injury ${ }^{18-24}$. Unfortunately, unlike animal models, clinical interventions designed to mitigate inflammation have uniformly failed to improve neurologic based outcomes in clinical trials. Likewise, inflammation-based interventions to improve outcomes using targeted and broad-based anti-inflammatory therapies do not improve outcomes in blunt trauma patients with $\mathrm{HS}^{25}$. Researchers have increasingly recognized how traumaassociated inflammation is enacted by a highly orchestrated network of immunologic mediators 5,26-30 and failure of immunomodulation to improve outcomes after TBI or blunt trauma reflects how traditional interventions do not affect the marked complexities of the immune response 13,26,28. Recently, blood purification by non-selective hemoadsorption (HA) has been introduced 
as an inflammation modulating intervention ${ }^{18,31-35}$. In contrast to pharmaceutical interventions, HA physically removes biologically active molecules from circulation and does not rely on interactions between an agent and cell surface receptors. HA reduced circulating concentrations of immunoactive molecules in patients with sepsis syndrome ${ }^{32,33,36-38}$ and when used in tandem with extracorporeal circulation in cardiopulmonary bypass surgery ${ }^{31,35}$ leading to improved outcomes in both patient populations. Little is known how HA reduces circulating concentrations of immunologic mediators and affects outcomes after trauma. In this study, we hypothesized that HA would reduce circulating levels of immunologic mediators in rats subjected to TBI and HS leading to improved clinical outcomes. The hypothesis was tested in rats, subjected to a combined injury including a controlled cortical impact (CCI) brain injury followed by HS. Rats were treated with either therapeutic or sham HA interventions. Our primary outcome was mortality by 96 hours after injury.

\section{Methods}

Experimental Protocol: All procedures were approved by the Indiana University School of Medicine IACUC. Two groups of Sprague Dawley male rats, 300 to 325 grams in weight, were tested. All rats were allowed seven days of free cage activity to acclimate to their surroundings with ad lib water and diet prior to testing. All rats were subjected to the same injury including TBI followed by HS. The experimental group was treated with HA at the conclusion of injury and the sham treatment group had sham circulation after injury. Following resuscitation from HS, the extracorporeal circuit was immediately established with either HA or sham treatment. Experimental and sham treatments lasted for 180 minutes based on previous studies using HA in rats ${ }^{36,37}$. Following sham or experimental treatment, animals were returned to individual cages and awakened from anesthesia. They were allowed free cage activity and ad lib diet. We analyzed 14 experimental and 19 sham rats in a 1:1 randomized design. The increased numbers 
of rats in the sham group resulted from greater numbers of death during sham treatment in an attempt to equalize numbers of rats surviving to 96 hours.

Closed Cortical Impact Model: TBI was induced using a CCI model. Rats were anesthetized via a nasal mask, and the rat's head was secured to a stereotaxic frame (David Kopf Instruments, Tujunga, CA). A 7-mm craniotomy was made between the lambda and bregma and centered over the right fronto-parietal cortex lateral to the central suture with dura intact. The impact device (Benchmark Stereotaxic Impactor; Myneurolab) struck the right fronto-parietal cortex with a velocity of $4.0 \mathrm{~m} / \mathrm{s}$, tissue deformation of $2.5 \mathrm{~mm}$, and impact duration of $100 \mathrm{~ms}$ with a $5-\mathrm{mm}$ impactor tip.

Hemorrhagic Shock Model: HS was established using a catheter inserted into the right femoral artery under loupe dissection. The artery was entered with a small intra-arterial catheter that was connected to a blood pressure transducer. A second catheter was surgically inserted using identical methods into the left femoral vein. Blood was removed at $0.5 \mathrm{ml} / \mathrm{minute}$ from the arterial catheter until systolic blood pressure was reduced to $40 \mathrm{mmHg}$ which was maintained for 60 minutes by further controlled hemorrhage or saline infusion into the venous catheter as dictated by ongoing blood pressure measurements. Subsequently, rats were resuscitated via autotransfusion of shed blood, augmented with normal saline if necessary, to restore the systolic blood pressure to $\geq 90 \mathrm{mmHg}$.

Hemoadsorption and Sham Treatment: Following HS, the rats were subsequently connected to an extracorporeal circuit to administer HA or sham treatment. The circuit included outflow from the right femoral artery catheter, passage through the treatment or sham cartridge, and return flow into the left femoral vein (Figure 1). The volume of the entire circuit was approximately $2.2 \mathrm{ml}$ of blood representing less than $10 \%$ of the rats circulating volume. In HA treatment animals, the circuit blood flowed through a $2.0 \mathrm{ml}$ cartridge that contained proprietary beads 
purpose designed to adsorb circulating molecules between 5.0 KDa and $60 \mathrm{KDa}$ (Cytosorbents; Monmouth Junction, NJ). In sham treatment animals, blood flowed through a $1.2 \mathrm{ml}$ canister without beads that was reduced in size to equalize the volume of blood within the circuit in sham and experimental animals. Following treatment, the tubing was removed and local hemostasis was obtained by direct pressure. Surgical wounds were closed with silk suture.

Blood Collection: Approximately $0.5 \mathrm{ml}$ of blood was aspirated from the venous catheter in K2EDTA-coated MiniCollect tube (Greiner Bio-One, Monroe, NC) immediately prior to injury (baseline), after injury but before the onset of sham or experimental treatment (0hr), at hourly intervals during experimental or sham treatment (1hr, $2 \mathrm{hr}$, and $3 \mathrm{hr}$ ), and at $24 \mathrm{hr}, 48 \mathrm{hr}$, and $96 \mathrm{hr}$ after injury from the tail vein. The plasma was isolated by centrifugation at 5,000 rpm for 10 min and was frozen at $-80^{\circ} \mathrm{C}$ until use. We measured 27 biomolecules using a multiplex panel (RECYMAG65K27PMX, Millipore, Billerica, MA) including interferon gamma (IFN $\gamma$ ), granulocyte colony stimulating factor (G-CSF), Eotaxin, Interleukin (IL)-2, IL-6, epidermal growth factor (EGF), Monocyte Chemotactic Protein-1 (MCP-1), interferon gamma induced protein 10 (IP-10), CXC motif ligand 1/keratinocyte derived cytokine/cytokine induced neutrophil chemoattractant $(\mathrm{GRO} / \mathrm{KC} / \mathrm{CINC}-1)$, granulocyte macrophage colony stimulating factor (GM-CSF), IL-1 $\alpha$, Leptin, IL-13, IL-10, IL-12p70, vascular endothelial growth factor (VEGF), Fractalkine, CXC motif ligand 5 (LIX), macrophage inflammatory protein 1 alpha (MIP-1 $\alpha$ ), IL-4, IL-1 $\beta$, IL-5, IL-17a, IL-18, MIP-2, Tumor Necrosis Factor Alpha (TNF $\alpha$ ), and chemokine ligand 5 (Rantes).

Histology: Three rats from both the sham and HA treatment groups that survived the experiment along with three control rats that had no surgery were anesthetized, perfused with PBS and fixed with 4\% paraformaldehyde in PBS. After being post fixed overnight, five sets of coronal sections containing the hippocampus were cut $(40 \mu \mathrm{m})$ with a vibratome (VT 1000; Leica, 
Nussloch, Germany) and collected in PBS. One set of sections was stained with standard hematoxylin and eosin (H\&E). The sections were mounted onto slides, air dried, dehydrated in graded series of ethanol, infiltrated in xylene, and embedded in paraffin. The slides were then examined with a microscope (BX50; Olympus, Tokyo, Japan). Images were acquired with a digital camera coupled to control software (DP70-BSW; Olympus) at $4 \mathrm{X}$ and $20 \mathrm{X}$ magnification.

Statistical analysis: Death times were recorded for all rats that did not survive. Survival functions were estimated using the product limit method and Kaplan-Meier curves were plotted. Time to death was compared between groups using the logrank test.

Two separate analyses were performed to evaluate biomarker concentrations. We compared experimental and sham treatment rats to evaluate treatment effects on mediator concentrations during treatment and after treatment. In addition, we compared survivor and non-survivor rats from the entire cohort of rats regardless of intervention to investigate biomarker changes that occurred after injury or any pre-injury differences in biomarkers that were associated with survival or death. Only one non-survivor lived past the $24 \mathrm{hr}$ time point, so the changes in biomarkers between survivor and non-survivor rats were analyzed from baseline through $3 \mathrm{hr}$ post-injury.

In both analyses, a random effects mixed model was used to assess levels of biomarkers during the three hour treatment time period. Of primary interest was the interaction term between group and time, assessing the change in circulating biomarkers over time between 1) sham and experimental groups; and 2) survivor and non-survivor rats from the entire cohort.. Available biomarker levels post-treatment were considered as repeated outcome measures, while baseline level was included in the model as a covariate. Since the sample sizes were modest, an unstructured covariance matrix was not used. Constant correlation between measurements was 
assumed and to minimize the number of parameters to be estimated, a compound symmetric covariance structure was used.

To determine if there were differences between the two comparison groups at individual time points during testing, we compared mean values at each time point between groups using t-tests. In addition, we compared mean values at individual time points during the treatment time period (1hr, 2hr, and 3hr) with the injury values (0hr) within each group using t-tests to determine longitudinal changes within each group during the treatment time frame. Finally, to determine residual effects of treatment on changes in mediators, biomarker values at $24 \mathrm{hr}, 48 \mathrm{hr}$, and $96 \mathrm{hr}$ were compared to baseline values in HA and sham treated rats using t-tests.

\section{Results}

Clinical Outcomes: Survival improved from 47\% (9 of 19 rats) in sham treated rats to $86 \%$ (12 of $14 ; \mathrm{p}=0.04$ ) in experimental rats. Nine of the ten sham treated rats died within 24 hours of injury. The two HA rats that died did so during HA treatment. Figure 2 depicts survival curves for HA and sham treated rats. Histologic evaluation demonstrated no differences in control, sham treated (untreated) and HA (treated) rats (Figure 3).

The Effect of HA on Circulating Concentrations of Biomarkers. HA decreased IL-2 throughout the entire treatment period and also decreased Eotaxin, IL-1 $\alpha$, IL-6, IL-13, MCP-1, and TNF $\alpha$ at discreet time points during the treatment period compared to sham treated animals. (Supplemental Figure 1). Seven of 27 biomarkers were elevated at one or more discreet time points during treatment ( $1 \mathrm{hr}$ to $3 \mathrm{hr}$ ) compared to the immediate post-injury (0hr) values, and no biomarkers were decreased during treatment in sham treated rats. In contrast, only three of 27 biomarkers were elevated at one or more discreet time points during treatment compared to postinjury values in HA rats, and IP-10, GM-CSF, Fractalkine and IL-18 were decreased at one or more discreet time points during treatment compared to post-injury values in HA rats 
(Supplemental Figure 1). Representative HA effects are selectively illustrated in temporal changes in GM-CSF, IL-6, IL-18, and TNF $\alpha$ (Figure 4). HA reduced concentrations of GM-CSF (at $3 \mathrm{hr}$ ) and IL-18 (at $1 \mathrm{hr}$ and $2 \mathrm{hr}$ ) compared to immediate post-injury concentrations. IL-6 (at 1hr) and TNFa (at 3hr) demonstrated decreased concentrations in HA treated rats compared to sham treated rats.

Six biomarkers remained elevated above baseline values at $24 \mathrm{hr}$ to $96 \mathrm{hr}$ after injury in sham rats. In contrast, during the same time period only two biomarkers were elevated above baseline values in HA rats (Supplemental Figure 1). MCP-1, IL-12p70, and IL-5 selectively illustrate differences in sham treatment and HA on changes in residual inflammation (Figure 5). During the treatment window, differences in concentrations in MCP-1, IL-12p70, and IL-5 between HA and sham rats were minimal. However at 24 and 48 hours after treatment, trends toward large increases in circulating concentrations of these molecules were measured in sham rats compared to HA rats (differences not statistically significant). In summary, HA resulted in decreases in circulating concentrations in several biomarkers compared to sham treatment, but the majority of cytokines were not affected by HA treatment.

Biomarker Concentrations in Survivor vs. Non-Survivor Rats: We investigated differences in circulating concentrations of biomarkers between survivor and non-survivor rats regardless of intervention to identify candidate biomarkers that were associated with death or survival. Thirteen of 27 cytokines were significantly $(\mathrm{p}<0.05)$ reduced in survivor rats over the entire three hour treatment period (IFN $\gamma$, EGF, VEGF, IP-10, GRO-KC-CINC1, Fractalkine, MIP-2, Rantes, Leptin, IL-2, IL-4, IL-6, IL-10, and IL-19) or increased (Leptin) compared to nonsurvivor rats (Supplemental Figure 2). Eotaxin, IL-1 $\alpha$, IL-4, IL-6, IL-17a, IL-18, MCP-1, GROKC-CINC, VEGF, and Fractalkine were all significantly reduced at one or more discreet time points from $0 \mathrm{hr}$ to $3 \mathrm{hr}$ in survivors compared to non-survivors. MIP-1 $\alpha$ was significantly 
elevated $3 \mathrm{hr}$ after injury in survivors compared to non-survivors. There were trends of increased IL-17a $(p=0.090)$, increased IFN $\gamma(p=0.087)$, and decreased Leptin $(p=0.070)$ at baseline prior to injury in non-survivors compared to survivors. Distinct biomarker patterns in survivors compared to non-survivors are selectively illustrated in Figure 6. EGF and IL-10 diverged within the first hour after injury between the survivors and non-survivors. In contrast, IFN $\gamma$ was higher in non-survivors even prior to injury and demonstrated little change during the treatment time period. Finally, IL-18 increased more in non-survivors compared to survivors immediately after the injury and prior to any treatment. In summary, temporal changes in biomarkers illustrate broad-based differences in circulating biomarker concentrations in surviving and nonsurviving rats.

\section{Discussion}

Our data support the hypothesis that HA of circulating blood improves survival in rats subjected to TBI and HS. HA improved survival from $47 \%$ to $86 \%$. HA reduced circulating concentrations of several cytokines, but it is likely that HA-mediated effects were caused by changing concentrations of molecules that were not quantified in this study. Only IL-2 and Leptin were significantly different in HA rats compared to sham rats throughout the entire treatment period and Leptin concentrations were actually increased in HA rats. In addition, HA resulted in reductions of only six of 27 biomarkers compared to sham treated rats at discreet time points during the treatment period. While HA reduced mortality, causation between changes in circulating concentrations of any of the measured biomarkers and survival is unproven. HA nonselectively reduces circulating concentrations of molecules in the $5 \mathrm{KDa}$ to $60 \mathrm{KDa}$ range which would include an expansive array of other bioactive molecules that could have affected outcomes in this experiment. Regardless of its mechanism of action, non-selective HA was highly effective in improving survival in rats subjected to TBI with HS. 
It is unknown how HA would affect outcomes in rats subjected to isolated TBI or isolated HS. In preliminary model development work, our rats routinely survived the CCI injury or HS performed in isolation. However, our survival rate was less than $50 \%$ when we combined the injuries during model development. Therefore, we chose to use the combined TBI/HS injury and focus on how treatment affected mortality. Histologic evaluation of the hippocampal region demonstrated no appreciable neuronal cell death in sham or treated rats indicating that the combined TBI/HS used in this model did not lead to neuronal cell death in unimpacted regions. It is possible that HA prevented neuronal death in brain tissue that was directly damaged by impact leading to improved survival. Likewise, it is possible that HA might improve other neurologic-based outcomes. However the effects of HA on injured brain tissue and on neurologic outcomes are unknown. Finally, the mechanisms by which HA prevented death in rats subjected to TBI and HS are not known.

Trauma incites an immunologically mediated response that typically is self-regulating and concentrations of circulating immunologic mediators return to baseline values as the injury resolves ${ }^{39}$. However, in some patients, immunologic homeostatic mechanisms fail and patients develop sustained elevated levels of inflammation which are associated with trauma-adverse phenotypes including multiple organ failure and nosocomial infections ${ }^{5,28}$. Detailed mechanistic structures of inflammation and progression of the immunologic response to injury continue to develop but are far from understood. The marked complexities and coordination of inflammation after injury are emerging as computational analyses have uncovered time-dependent and spatial networks of immunologic orchestration ${ }^{5,26,27,40-42}$. Our data illustrated some intricacies of immunologic orchestration. For example, several of the mediators such as G-CSF and MCP-1 (Supplemental Figure 1) were minimally affected by injury or HA treatment, but were notably greater in sham rats $24 \mathrm{hr}$ to $48 \mathrm{hr}$ after injury. Likewise other mediators such as VEGF, 
Fractalkine, MIP-1 $\alpha$, and IL-13 were all decreased by HA during treatment but $24 \mathrm{hr}$ later were unchanged compared to sham controls. These observations align with contemporary models of the immunologic response to injury which increasingly describe a complex molecular orchestration of pro-inflammatory and anti-inflammatory signals $26,27,41$. Computational analyses are necessary to decipher how interventions such as HA affect immunologic orchestration and affect outcomes after injury.

The data from this experiment suggest that immunologic response differences between specimens likely affect outcomes. For example, there were trends toward higher IFN $\gamma$, higher IL-17a and lower Leptin in non-survivors at baseline prior to any injury (Supplemental Figure 2). Likewise, there were trends toward greater injury-associated increases in IL-18 and Fractalkine in non-survivors compared to survivors at $0 \mathrm{hr}$ prior to any intervention. Finally, nearly half of the biomarkers were different during the initial three hour time period after injury in survivors compared to non-survivors (Supplemental Figure 2). Further investigation will be necessary to understand how individual differences in immune response affect outcomes.

Inflammation mitigation to improve outcomes after TBI has been tested extensively in animal models. Bergold thoroughly summarized results from 95 distinct animal trials of a variety of anti-inflammatory interventions to improve outcomes after TBI ${ }^{17}$. Collectively, these results unambiguously demonstrate that inflammation exacerbates TBI and that inflammation mitigation can reduce secondary brain injury. In addition, reducing secondary brain injury consistently improved histologic and phenotypic outcomes in specimens with inflammation blockade. However, none of these studies quantified treatment effects on mortality. In addition, while animal evidence has demonstrated consistent improvements in a variety of molecular, histologic, motor, and cognitive outcomes, similar inflammation-mitigation interventions in human trials have uniformly failed to improve outcomes after TBI ${ }^{18-24,43}$. These trials have included broad- 
based corticosteroid interventions ${ }^{18-20,24}$ and targeted interventions that block potent cytokines including TNF $\alpha$ and IL-1 ${ }^{23,43}$. Consensus amongst authorities is that immunologic-based interventions have failed to affect the broader complexities of temporal and spatial orchestration of the immune response to injury leading to clinical failure ${ }^{13}$.

HA has a unique mechanism of action that physically removes molecules from circulation. It is effective at removing cytokines as many of these molecules are within the $5 \mathrm{KDa}$ to $60 \mathrm{KDa}$ purification envelope of Cytosorbents. In contrast to traditional pharmaceuticals, HA does not mediate its effects by interacting with biologically active cell surface receptors. It is possible that traditional pharmaceutical mechanisms (cell surface receptor blockade, cell surface receptor stimulation, nuclear transcription effects) may be altered by injury and that a simplified approach that does not rely on any interaction with cells offers an effective immunomodulation strategy. HA has been shown to improve outcomes in a variety of animal models and in clinical applications. Zhang and colleagues used HA in rats subjected to sepsis through cecal puncture ${ }^{38}$. In this model, the investigators demonstrated modest reductions in several cytokines including decreases in IL6 similar to what we measured in this experiment. The investigators demonstrated modest improvements in mortality from $50 \%$ in untreated animals to $26 \%$ in treated animals. These data parallel previous small animal experimentation by the same research group that HA reduces multiple mediators during treatment and on a delayed basis $36,37,44$. Clinically, in 27 patients who developed septic shock with kidney failure, HA improved hemodynamic parameters, reduced blood lactate levels, and reduced predicted mortality rates. Effects were most pronounced in patients treated within 24 hours of developing kidney failure ${ }^{33}$. Trager and colleagues reported reductions in cytokines and improved outcomes in a retrospective series of 16 patients who developed SIRS after cardiopulmonary bypass ${ }^{35}$. Recently in a randomized controlled clinical trial, it was shown that HA during surgery reduced circulating 
levels of IL-10 in patients placed on cardiac bypass ${ }^{31}$. However, changes in circulating IL-10 did not extrapolate into any clinical improvement. Likewise in several other clinical trials, the effect of HA on phenotypic improvement are encouraging but not proven ${ }^{18,32,34,45}$.

There are multiple potential sources of error in our experiment. The entire experimental setup is technically demanding and achieving uniform surgical access to homogenize the brain injury, produce consistent HS, normalize resuscitation, and produce consistent HA was challenging. Fortunately, we had performed these procedures for several other protocols and had become proficient with our surgical methods prior to embarking on this protocol. The cause of death in rats was unknown in this experiment. The high mortality rate can be safely assumed to result from the combined injury as we observed minimal mortality in rats subjected to either isolated CCI or HS in model development. In addition, surviving rats in both sham and treatment groups resumed presumably normal motor and feeding behavior consistent with other protocols using similar or identical CCI parameters inducing brain injury ${ }^{17}$. This suggests the magnitude of the TBI was moderate, but the TBI magnitude was not formally quantified in this experiment. It is unknown how HA prevented death in our model and HA reductions in mortality may have been independent from preventing propagation of TBI. For example, it is possible that HA prevented death by reducing the combined effects of TBI and HS on cardiovascular function. We only quantified changes in mortality and we did not quantify motor-based or functional outcomes in rats that survived. Therefore, we do not know what the effects of HA are on longer-term neurological outcomes after TBI. We measured an expanded panel of cytokines felt to reflect a trauma-relevant menu of immunologic mediators. However, HA non-selectively adsorbs molecules in the $5 \mathrm{KDa}$ to $60 \mathrm{KDa}$ size range and it is distinctly possible that improvements in survival were affected by changes in other molecules that we did not quantify. Additionally, it was anticipated that HA would result in broad reductions in circulating immunologically active 
molecules and it is not surprising that there were general increases in several of the mediators in non-surviving rats compared to survivors. While the molecules we measured have been integrated into a multiplex panel relevant to trauma and shock, it is unknown how changes in any individual molecule affected survival. However, the data do provide some foundation and direction for more in-depth mechanistic studies pertaining to immunologically mediated outcomes after injury.

In conclusion, blood purification by HA reduced circulating concentrations of multiple cytokines in rats subjected to combined TBI and HS. HA increased survival from $47 \%$ in sham treated rats to $86 \%$ in experimentally treated rats. HA physically removes biologically active molecules from circulation which is distinct from pharmaceutical inflammation-mitigation interventions that block cell surface receptors, modulate transcription and modulate translation. HA has been safe and effective in human trials and offers a new alternative to be investigated to improve outcomes in patients with TBI and HS.

Author Contribution: TOM designed the study, developed the rat model, participated in data collection, data analysis and interpretation and was the lead author in drafting the manuscript; ZL was the lead surgeon for the study and participated in model development, data acquisition, data analysis and manuscript review; YK assisted on all surgeries and participated in data acquisition, data analysis and interpretation and manuscript review; FAW participated in data analysis and interpretation and manuscript preparation and review; ZS participated in data acquisition, data analysis, data interpretation and manuscript review; FW participated in data acquisition, data analysis, data interpretation and manuscript review; ZCX participated in model development, data analysis, data interpretation and manuscript review; RBR designed the study participated in model development, data acquisition, data interpretation and was the co-lead in drafting the manuscript. 


\section{References}

1. Hariri RJ, Firlick AD, Shepard SR, Cohen DS, Barie PS, Emery JM $3^{\text {rd }}$, Ghajar JB. Traumatic brain injury, hemorrhagic shock, and fluid resuscitation: effects on intracranial pressure and brain compliance. J Neurosurg. Sep 1993;79(3):421-427.

2. Maegele M. Coagulopathy after traumatic brain injury: incidence, pathogenesis, and treatment options. Transfusion. Jan 2013;53 Suppl 1:28S-37S.

3. Corral L, Javierre CF, Ventura JL, Marcos P, Herrero JI, Manez R. Impact of nonneurological complications in severe traumatic brain injury outcome. Crit Care. 2012;16(2):R44. 4. Lou M, Chen X, Wang K, Xue Y, Cui D, Xue F. Increased intracranial pressure is associated with the development of acute lung injury following severe traumatic brain injury. Clin Neurol Neurosurg. Jul 2013;115(7):904-908.

5. Namas RA, Vodovotz Y, Almahmoud K, Abdul-Malak O, Zaaqoq A, Namas R, Mi Q, Barclay D, Zuckerbraun B, Peitzman AB, et al. Temporal Patterns of Circulating Inflammation Biomarker Networks Differentiate Susceptibility to Nosocomial Infection Following Blunt Trauma in Humans. Ann Surg. Nov 32014.

6. Rincon F, Ghosh S, Dey S, Maltenfort M, Vibbert M, Urtecho J, McBride W, Moussouttas M, Bell R, Ratliff JK, et al. Impact of acute lung injury and acute respiratory distress syndrome after traumatic brain injury in the United States. Neurosurg. Oct 2012;71(4):795-803.

7. Das M, Mohapatra S, Mohapatra SS. New perspectives on central and peripheral immune responses to acute traumatic brain injury. J Neuroinflammation. Oct 12 2012;9:236.

8. Stein DM, Lindell AL, Murdock KR, Kufera JA, Menaker J, Bochicchio GV, Arabi B, Scalea TM. Use of serum biomarkers to predict cerebral hypoxia after severe traumatic brain injury. J Neurotrauma. Apr 10 2012;29(6):1140-1149. 
9. Kelley BJ, Lifshitz J, Povlishock JT. Neuroinflammatory responses after experimental diffuse traumatic brain injury. J Neuropathol Exp Neurol. Nov 2007;66(11):989-1001.

10. Szmydynger-Chodobska J, Strazielle N, Gandy JR, Keefe TH, Zink BJ, Ghersi-Egea JF, Chodobski A. Posttraumatic invasion of monocytes across the blood-cerebrospinal fluid barrier. Journal of cerebral blood flow and metabolism :J Cereb Blood Flow Metab. Jan 2012;32(1):93104.

11. Lee K, Rincon F. Pulmonary complications in patients with severe brain injury. Crit Care Res Pract. 2012;2012:207247.

12. Finnie JW. Neuroinflammation: beneficial and detrimental effects after traumatic brain injury. Inflammopharmacology. Aug 2013;21(4):309-320.

13. Lozano D, Gonzales-Portillo GS, Acosta S, De la Pena I, Tajiri N, Kaneko Y, Borlongan $\mathrm{CV}$. Neuroinflammatory responses to traumatic brain injury: etiology, clinical consequences, and therapeutic opportunities. Neuropsychiatr Dis Treat. 2015;11:97-106.

14. Bonds BW, Yang S, Hu PF, Kalpakis K, Stansbury LG, Scalea TM, Stein DM. Predicting secondary insults after severe traumatic brain injury. J Trauma Acute Care Surg. Jul 2015;79(1):85-90; discussion 90.

15. Lo TY, Jones PA, Minns RA. Combining coma score and serum biomarker levels to predict unfavorable outcome following childhood brain trauma. $J$ Neurotrauma. Dec 2010;27(12):2139-2145.

16. Stein DM, Lindel AL, Murdock KR, Kufera JA, Menaker J, Scalea TM. Use of serum biomarkers to predict secondary insults following severe traumatic brain injury. Shock. Jun 2012;37(6):563-568.

17. Bergold PJ. Treatment of traumatic brain injury with anti-inflammatory drugs. Exp Neurol. Jan 2016;275 Pt 3:367-380. 
18. Didar TF, Cartwright MJ, Rottman M, Graveline AR, Gamini N, Watters AL, Leslie DC, Mammoto T, Rodas MJ, Kang JH, et al. Improved treatment of systemic blood infections using antibiotics with extracorporeal opsonin hemoadsorption. Biomaterials. Oct 2015;67:382-392.

19. Edwards P, Arango M, Balica L, Cottingham R, El-Sayed H, Farrell B, Fernandes J, Gogichaisvili T, Golden N, Hartzenberg B, et. al. Final results of MRC CRASH, a randomised placebo-controlled trial of intravenous corticosteroid in adults with head injury-outcomes at 6 months. Lancet. Jun 4-10 2005;365(9475):1957-1959.

20. Roberts I, Yates D, Sandercock P, Farrell B, Wasserberg J, Lomas G, Cottingham R, Svoboda P, Brayley N, Mazairac G, et al. Effect of intravenous corticosteroids on death within 14 days in 10008 adults with clinically significant head injury (MRC CRASH trial): randomised placebo-controlled trial. Lancet. Oct 9-15 2004;364(9442):1321-1328.

21. Robertson CS, Hannay HJ, Yamal JM, Gopinath S, Goodman JC, Tilley BC, Epo Severe TBI Trial Investigators, Baldwin A, Rivera Lara L, Saucedo-Crespo H, et al. Effect of erythropoietin and transfusion threshold on neurological recovery after traumatic brain injury: a randomized clinical trial. JAMA. Jul 2 2014;312(1):36-47.

22. Tapia-Perez J, Sanchez-Aguilar M, Torres-Corzo JG, Gordillo-Moscoso A, MartinezPerez P, Madeville P, de la Cruz-Mendoza E, Chalita-Williams J. Effect of rosuvastatin on amnesia and disorientation after traumatic brain injury (NCT003229758). J Neurotrauma. Aug 2008;25(8):1011-1017.

23. Tobinick E, Kim NM, Reyzin G, Rodriguez-Romanacce H, DePuy V. Selective TNF inhibition for chronic stroke and traumatic brain injury: an observational study involving 629 consecutive patients treated with perispinal etanercept. CNS drugs. Dec 2012;26(12):1051-1070. 
24. Wright DW, Yeatts SD, Silbergleit R, Palesch YY, Hertzberg VS, Frankel M, Goldstein FC, Caveney AF, Howlett-Smith H, Bengelink EM, et al. Very early administration of progesterone for acute traumatic brain injury. N Engl J Med. Dec 25 2014;371(26):2457-2466.

25. Spruijt NE, Visser T, Leenen LP. A systematic review of randomized controlled trials exploring the effect of immunomodulative interventions on infection, organ failure, and mortality in trauma patients. Crit Care. 2010;14(4):R150.

26. Almahmoud K, Namas RA, Abdul-Malak O, Zaaqoq AM, Zamora AM, Zuckerbraun BS, Sperry J, Peitzman AB, Billiar TR, Vodovotz Y. Impact of Injury Severity on Dynamic Inflammation Networks Following Blunt Trauma. Shock. Aug 2015;44(2):101-109.

27. An G, Nieman G, Vodovotz Y. Toward computational identification of multiscale "tipping points" in acute inflammation and multiple organ failure. Ann Biomed Eng. Nov 2012;40(11):2414-2424.

28. Namas RA, Almahmoud K, Mi Q, Ghuma A, Namas R, Zaaqoq A, Zhu X, Abdul-Malak O, Sperry J, Zamora R, et al. Individual-specific principal component analysis of circulating inflammatory mediators predicts early organ dysfunction in trauma patients. $J$ Crit Care. Dec 2016;36:146-153.

29. Schimunek L, Namas RA, Yin J, Liu D, Barclay D, El-Dehaibi F, Abboud A, Lindberg H, Zamora R, Billiar TR, et al. An Enrichment Strategy Yields Seven Novel Single Nucleotide Polymorphisms Associated With Mortality and Altered Th17 Responses Following Blunt Trauma. Shock. Mar 2018;49(3):259-268.

30. Vodovotz Y, Constantine G, Faeder J, Mi Q, Rubin J, Bartels J, Sarkar J, Squires RH Jr, Okonkwo DO, Gerlach J, et al. Translational systems approaches to the biology of inflammation and healing. Immunopharmacol Immunotoxicol. Jun 2010;32(2):181-195. 
31. Bernardi MH, Rinoesl H, Dragosits K, Ristl R, Hoffelner F, Opfermann P, Lamm C, Preissing F, Wiedemann D, Hiesmayr MJ, et al. Effect of hemoadsorption during cardiopulmonary bypass surgery - a blinded, randomized, controlled pilot study using a novel adsorbent. Crit Care. Apr 09 2016;20:96.

32. Bruenger F, Kizner L, Weile J, Morshuis M, Gummert JF. First successful combination of ECMO with cytokine removal therapy in cardiogenic septic shock: a case report. Int J Artif Organs. Feb 2015;38(2):113-116.

33. Kogelmann K, Jarczak D, Scheller M, Druner M. Hemoadsorption by CytoSorb in septic patients: a case series. Crit Care. Mar 27 2017;21(1):74.

34. Tomescu DR, Olimpia Dima S, Ungureanu D, Popescu M, Tulbure D, Popescu I. First report of cytokine removal using $\operatorname{CytoSorb}(\mathrm{R})$ in severe noninfectious inflammatory syndrome after liver transplantation. Int J Artif Organs. May 16 2016;39(3):136-140.

35. Trager K, Fritzler D, Fischer G, Schroder J, Skrabal C, Liebold A, Reinelt H. Treatment of post-cardiopulmonary bypass SIRS by hemoadsorption: a case series. Int J Artif Organs. May $162016 ; 39(3): 141-146$.

36. Kellum JA, Song M, Venkataraman R. Hemoadsorption removes tumor necrosis factor, interleukin-6, and interleukin-10, reduces nuclear factor-kappaB DNA binding, and improves short-term survival in lethal endotoxemia. Crit Care Med. Mar 2004;32(3):801-805.

37. Peng ZY, Carter MJ, Kellum JA. Effects of hemoadsorption on cytokine removal and short-term survival in septic rats. Crit Care Med. May 2008;36(5):1573-1577.

38. Zhang J, Peng Z, Maberry D, Volpe J, Kimmel JD, Federspiel WJ, Kellum JA. Effects of hemoadsorption with a novel adsorbent on sepsis: in vivo and in vitro study. Blood Purif. 2015;39(1-3):239-245. 
39. Sauaia A, Moore FA, Moore EE. Postinjury Inflammation and Organ Dysfunction. Crit Care Clin. Jan 2017;33(1):167-191.

40. Abboud A, Namas RA, Ramadan M, Mi Q, Almahmoud K, Abdul-Malak O, Azhar N, Zaaqoq A, Namas R, Barclay DA, et al. Computational Analysis Supports an Early, Type 17 Cell-Associated Divergence of Blunt Trauma Survival and Mortality. Crit Care Med. Nov 2016;44(11):e1074-e1081.

41. Abdul-Malak O, Vodovotz Y, Zaaqoq A, Guardado J, Almahmoud K, Yin J, Zuckerbraun B, Peitzman AB, Sperry J, Billiar TR, et al. Elevated Admission Base Deficit Is Associated with a Complex Dynamic Network of Systemic Inflammation Which Drives Clinical Trajectories in Blunt Trauma Patients. Mediators Inflamm. 2016;2016:7950374.

42. Namas RA, Bartels J, Hoffman R, Barclay D, Billiar TR, Zamora R, Vodovotz Y. Combined in silico, in vivo, and in vitro studies shed insights into the acute inflammatory response in middle-aged mice. PloS one. 2013;8(7):e67419.

43. Helmy A, Guilfoyle MR, Carpenter KL, Pickard JD, Menon DK, Hutchinson PJ. Recombinant human interleukin-1 receptor antagonist in severe traumatic brain injury: a phase II randomized control trial. J Cereb Blood Flow Metab. May 2014;34(5):845-851.

44. Namas RA, Namas R, Lagoa C, Barclay D, Mi Q, Zamora R, Peng Z, Wen X, Fedorchak MV, Valenti IE, et al. Hemoadsorption reprograms inflammation in experimental gram-negative septic peritonitis: insights from in vivo and in silico studies. Mol Med. Dec 20 2012;18:13661374.

45. Rimmele T, Kaynar AM, McLaughlin JN, Bishop JV, Fedorchak MV, Chuasuwan A, Peng Z, Singbartl K, Frederick DR, Zhu L, et al. Leukocyte capture and modulation of cellmediated immunity during human sepsis: an ex vivo study. Crit Care. Mar 26 2013;17(2):R59. 


\section{Figure Legends}

Figure 1. Experimental setup for sham treatment and hemoadsorption (HA). The femoral artery catheter (FA) is in series with the cartridge (CYT). In HA treated rats, a $2.0 \mathrm{ml}$ cartridge contained proprietary beads (Cytosorbents; Monmouth Junction, NJ) that removed circulating molecules in the $5 \mathrm{KDa}$ to $60 \mathrm{KDa}$ range. In sham rats, the cartridge was empty and reduced to $1.2 \mathrm{ml}$ to equalize the volume of blood in the circuit in both groups. Outflow rate from the cartridge was maintained with a pump and returned to the femoral vein catheter (FV).

Figure 2. Survival curves for experimental rats treated with HA compared to sham treated rats demonstrates efficacy of HA treatment to prevent death. Two of $14 \mathrm{HA}$ rats died and did so early after injury during HA treatment. Ten of 19 sham treated rats died with only one rat surviving beyond $24 \mathrm{hr}$ after injury.

Figure 3. Representative Hematoxylin and Eosin stained hippocampal sections from uninjured rats (Control), sham-treated (Untreated), and HA-treated (Treated) demonstrated no fundamental differences in cell death or inflammatory composition in the sham-treated compared to HAtreated rats. Higher magnification shows similar cell viability and inflammatory changes in the Cornu Ammonis (CA)1, CA3, and Dentate Gyrus regions.

Figure 4. Four purposefully selected biomarkers demonstrating how HA (dashed lines) affected circulating concentrations of immunoactive molecules compared to sham rats (solid line). An single asterisk $(*)$ denotes statistically significant difference in biomarker concentration $(\mathrm{p}<$ 0.05) in HA treated rats compared to sham treated rats at that specific time point; ** denotes significantly different biomarker concentration $(\mathrm{p}<0.05)$ at that specific time point compared to 
the $0 \mathrm{hr}$ value in HA treated rats; \#\# denotes significantly different biomarker concentration $(\mathrm{p}<$ 0.05 ) at that specific time point compared to $0 \mathrm{hr}$ value in sham treated rats. HA reduced GMCSF and IL-18 (**) during the treatment time period compared to immediate post-injury values at $0 \mathrm{hr}$. In contrast, in sham treated rats, GM-CSF, IL-6, and TNF- $\alpha$ were significantly increased in concentration during the treatment period (\#\#) compared to immediate post-injury values at 0hr. Concentrations of GM-CSF at 96hr, IL-6 at $1 \mathrm{hr}$ and TNF- $\alpha$ at $3 \mathrm{hr}$ were lower in HA treated rats compared to sham treated rats $\left(^{*}\right)$. Finally, the injury caused a significant increase in IL-18 in both sham treated rats (\#\#) and HA treated rats (**) at 0 hr compared to baseline values.

Figure 5. Changes in biomarker concentrations in MCP-1, IL-5, and IL-12p70 selectively illustrate three mediators that had relatively minimal change in concentration after injury or during treatment but had different trajectories during the subsequent $24 \mathrm{hr}$ to $96 \mathrm{hr}$ after injury in HA treated rats (dashed lines) compared to sham treated rats (solid lines). These three mediators demonstrated trends toward greater increases during the $24 \mathrm{hr}$ to $96 \mathrm{hr}$ time frame in sham treated rats compared to HA treated rats. MCP-1 concentrations were greater at $24 \mathrm{hr}, 48 \mathrm{hr}$, and $96 \mathrm{hr}$ in sham (\#\#\#) and HA (***) treated rats compared to baseline values with trends toward greater increases in sham treated rats. Likewise, IL-5 was increased over baseline values at $48 \mathrm{hr}$ and 96hr after injury in sham treated rats $(\# \#)$ with a trend $(\mathrm{p}=0.088)$ toward being greater than HA treated rats at $48 \mathrm{hr}$ after injury. Finally, IL-12p70 trended higher in sham treated rats at $24 \mathrm{hr}(\mathrm{p}$ $=0.091)$ and $48 \mathrm{hr}(\mathrm{p}=0.104)$ compared to HA treated rats. 
Figure 6. Selected data demonstrate that there were distinctive biomarker patterns that occurred immediately after injury (0hr) and during the treatment time period (0hr $-3 \mathrm{hr})$ in survivors (dashed lines) compared to non-survivors (solid lines) drawn from the entire cohort of rats regardless of treatment. EGF, IL-18, IL-10, and IFN $\gamma$ illustrate four mediators that were significantly increased in non-survivors compared to survivors over the entire treatment period (horizontal bar with associated $\mathrm{p}$ value above data points). Distinctive patterns are illustrated within these four biomarkers. EGF and IL-10 had similar baseline and post-injury (0hr) values in survivors and non-survivors, but diverged in magnitude after the injury and during the three hour treatment time frame. In contrast, IL-18 diverged between survivors and non-survivors after the injury. Finally, IFN $\gamma$ was seemingly different between survivors and non-survivors at baseline, immediately after injury and during the treatment time frame.

Supplemental Figure 1. Time-dependent changes in all 27 biomarkers in HA treated rats $(n=14$; dashed lines) and sham treated rats $(\mathrm{n}=19$; solid lines). A single asterisk $(*)$ denotes a significant difference $(\mathrm{p}<0.05)$ between sham rats and HA rats at an individual time point (i.e. IL- $1 \alpha$ at the 1 hr time point). In HA treated rats, $* *$ denotes a statistically significant decrease or increase in biomarker concentration $(\mathrm{p}<0.05)$ at the denoted time point compared to the biomarker concentration in the HA group at the immediate post-injury Ohr time point. Likewise, in sham treated rats, \#\# denotes a statistically significant decrease or increase in biomarker concentration $(\mathrm{p}<0.05)$ at the denoted time point compared to the biomarker concentration in the sham group at the post-injury $0 \mathrm{hr}$ time point. A triplicate symbol of *** in HA rats or \#\#\# in sham rats denotes a statistically significant increase or decrease in a biomarker concentration (p $<0.05$ ) at the $24 \mathrm{hr}, 48 \mathrm{hr}$, or $96 \mathrm{hr}$ time point compared to baseline values of the biomarker within each group. These comparisons were conducted to identify residual changes in biomarker 
concentrations in sham or treatment groups. Finally in Leptin and IL-2, the horizontal bar above the data points and associated p-value denotes statistically significant differences in biomarker concentration between sham and HA rats over the entire treatment period ( $0 \mathrm{hr}$ to $3 \mathrm{hr}$ ). There was a trend toward a difference over the treatment time period in GM-CSF $(p=0.068)$ and IP-10 $(\mathrm{p}=0.068)$.

Supplemental Figure 2. Time-dependent changes in all 27 biomarkers comparing rats that either survived to $96 \mathrm{hr}(\mathrm{n}=21$; dashed lines) or died prior to the $96 \mathrm{hr}$ time point $(\mathrm{n}=12$; solid lines $)$ from the entire cohort of rats regardless of intervention. Note of the 12 rats that died, 11 died prior to the $24 \mathrm{hr}$ time point so biomarkers in this figure are presented only until the $3 \mathrm{hr}$ time point. Biomarkers denoted by a single asterisk $\left(^{*}\right)$ are significantly different in concentration (p $<0.05$ ) between survivors and non-survivors at that time point. Biomarkers denoted by either \#\# (non-survivors) or $* *$ (survivors) are significantly different $(\mathrm{p}<0.05)$ compared to the $0 \mathrm{hr}$ value within the same group at the denoted time point (i.e. IL-4 is significantly higher in non-survivors at $1 \mathrm{hr}$ and $3 \mathrm{hr}$ compared to the $0 \mathrm{hr}$ value in non-survivors). A horizontal bar either above or below the $0 \mathrm{hr}$ to $3 \mathrm{hr}$ data with an associated $\mathrm{p}$ value denotes that biomarker concentrations were significantly different $(\mathrm{p}<0.05)$ over the entire treatment time period in non-survivors compared to survivors. Fourteen of the 27 biomarkers were significantly different over the entire treatment period in survivors compared to non-survivors. 
Figure 1

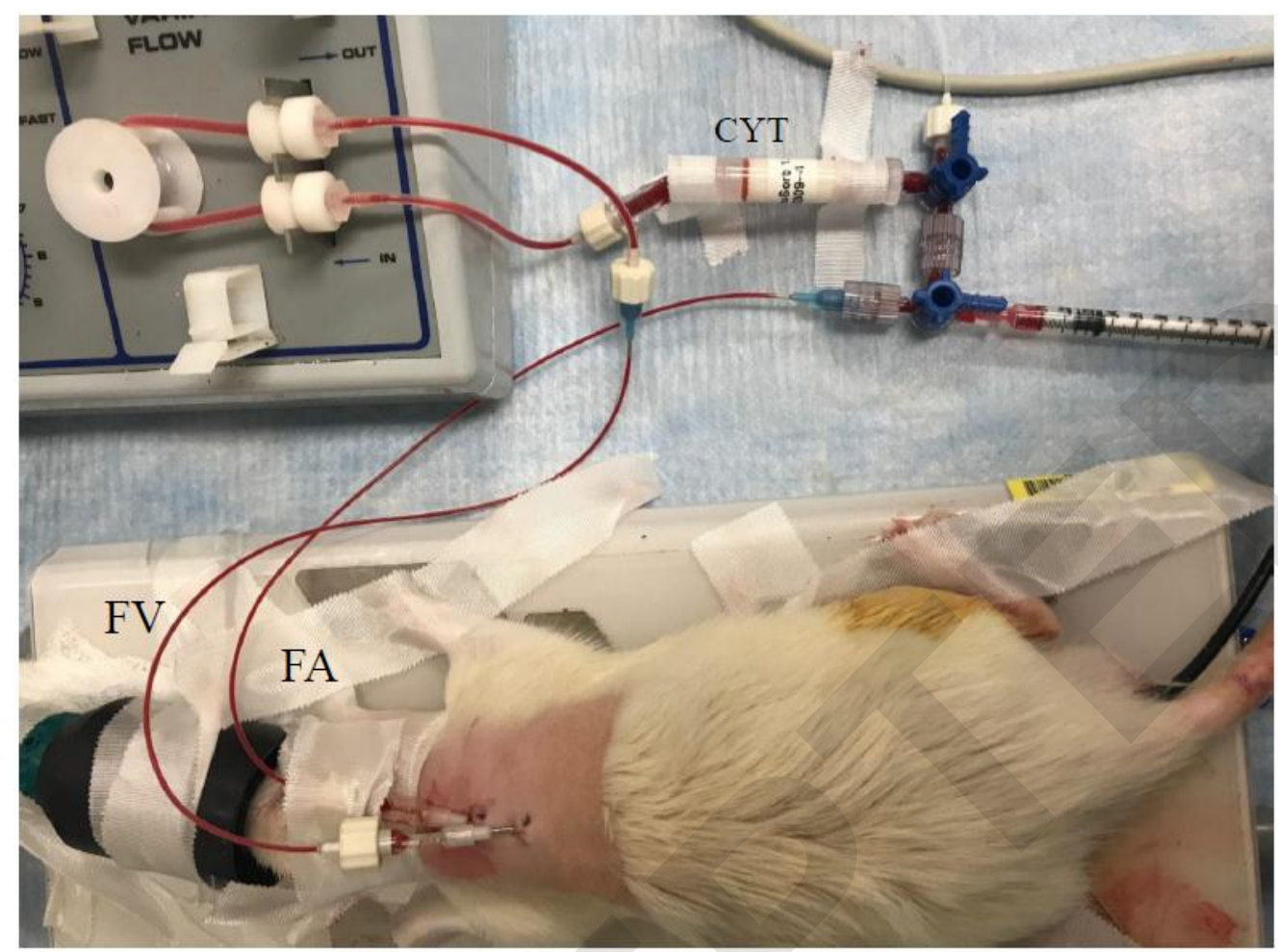


Figure 2

\section{Survival Post Treatment}

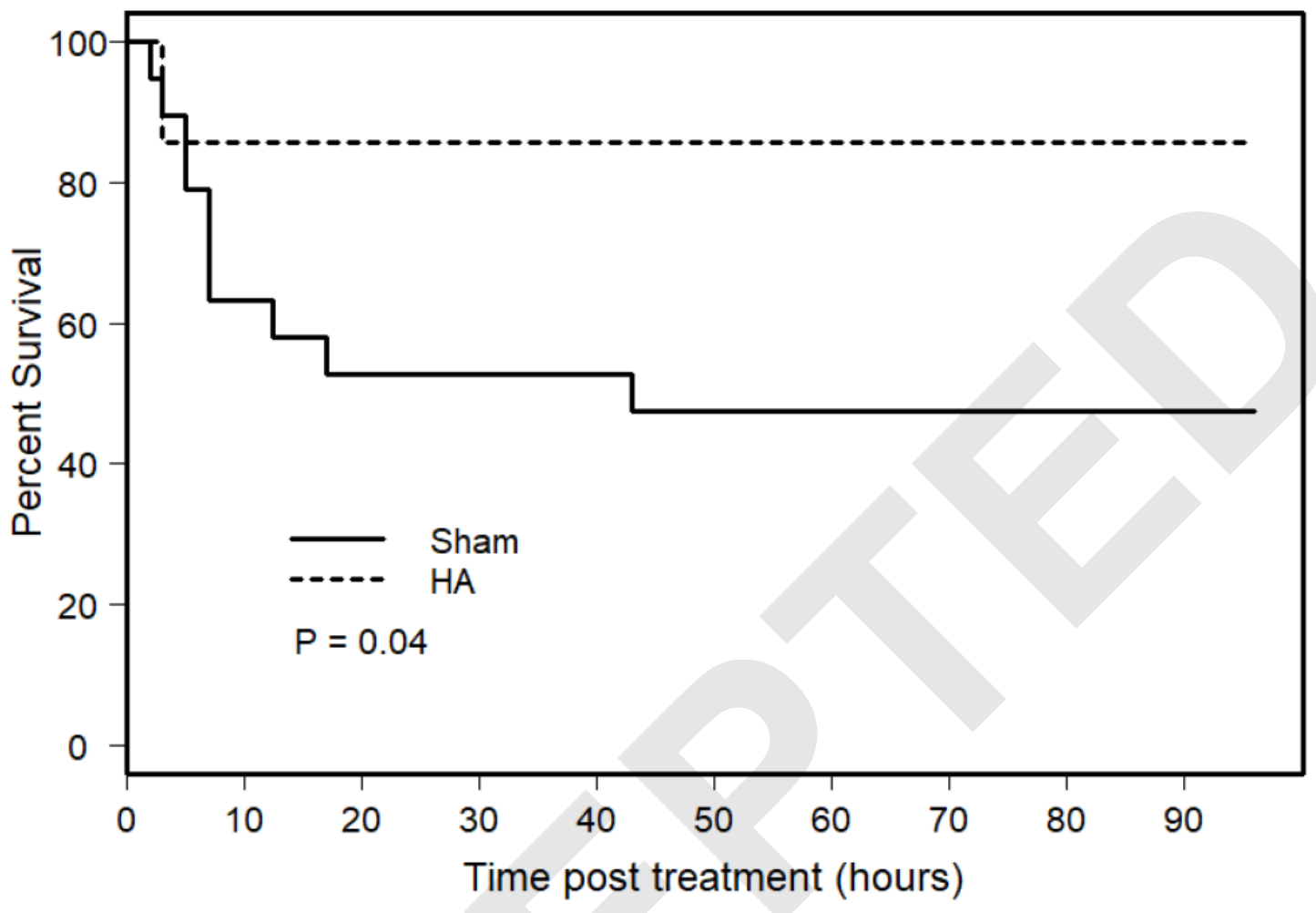


Figure 3

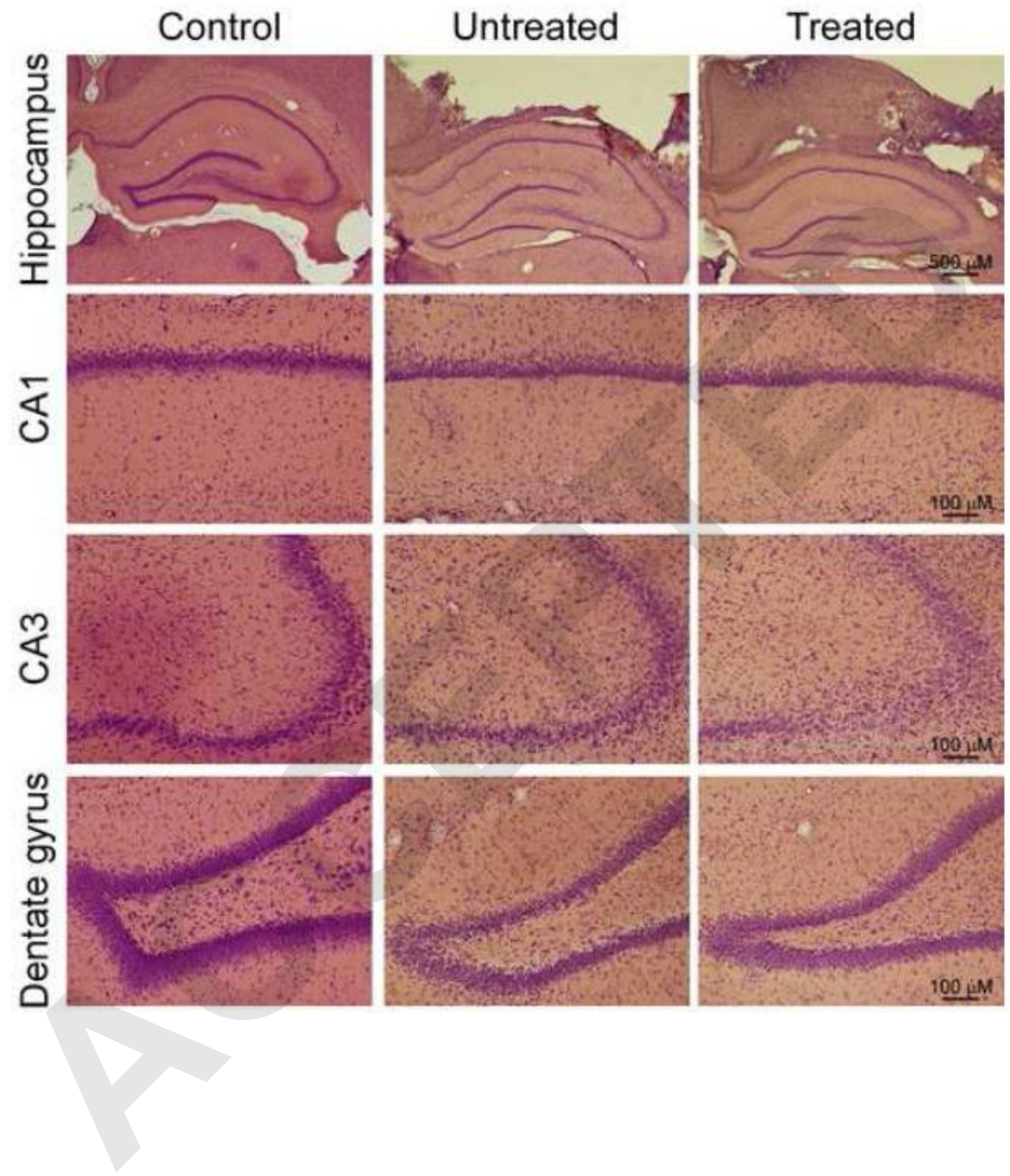




\section{Figure 4}
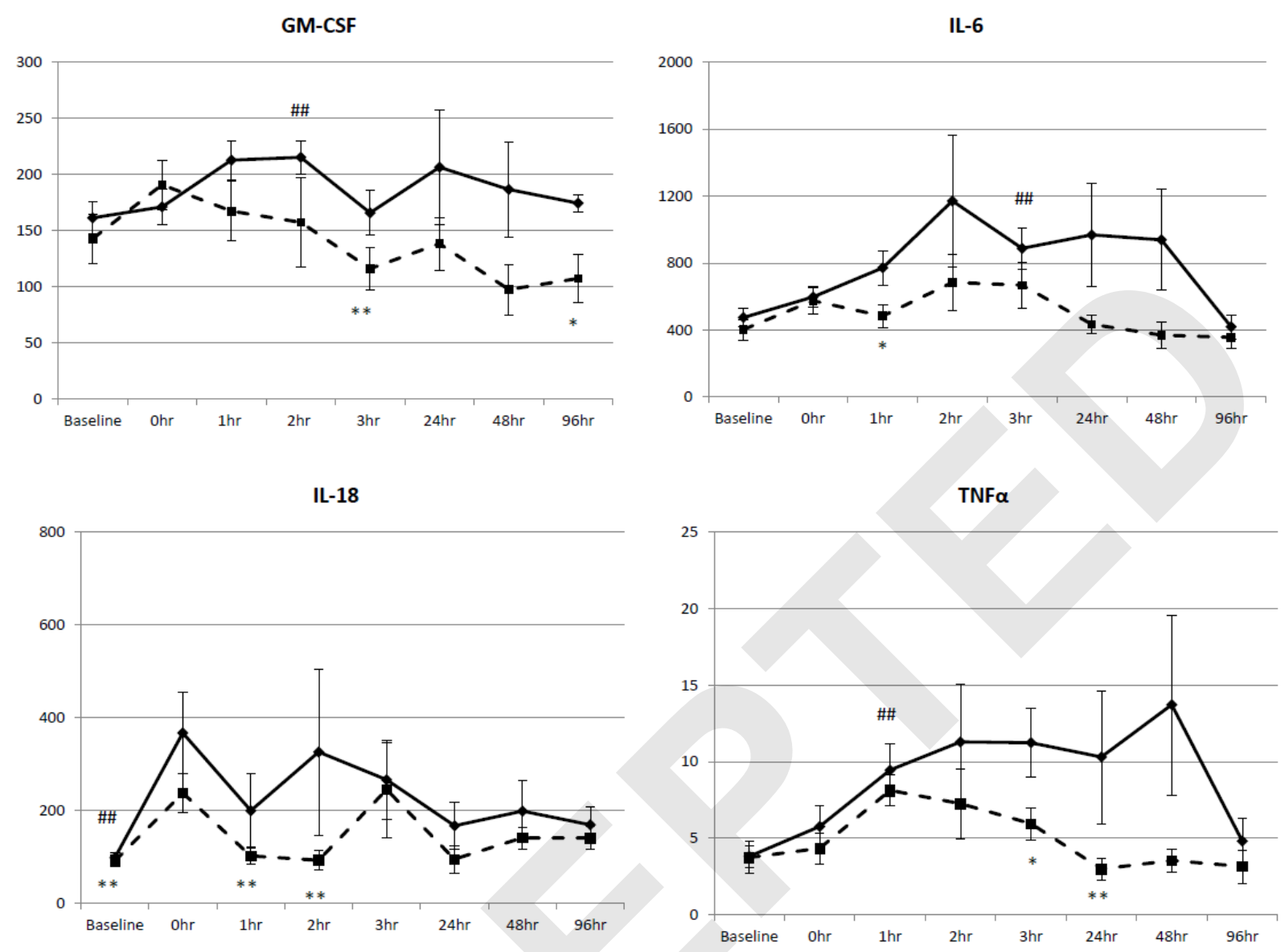

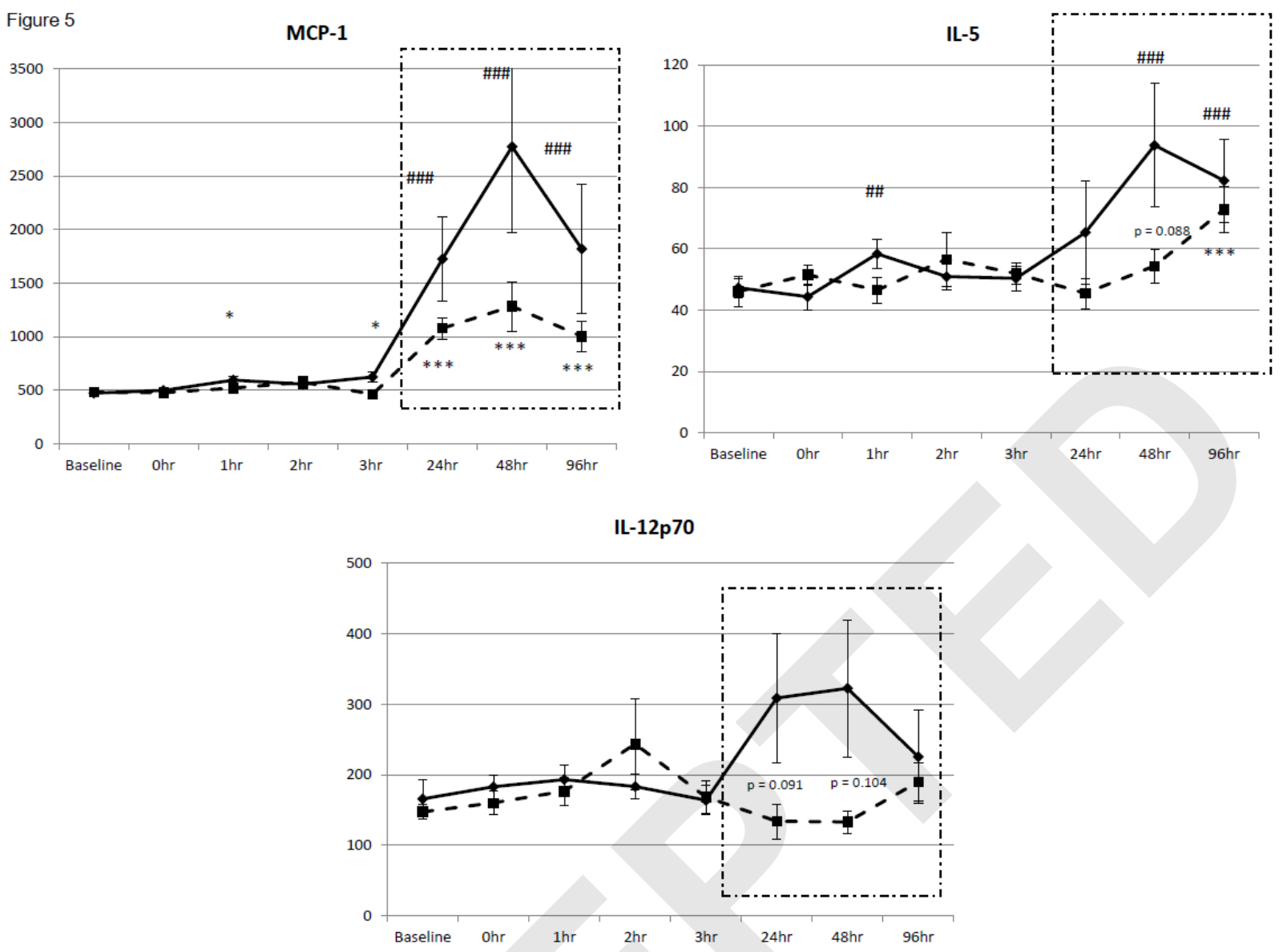


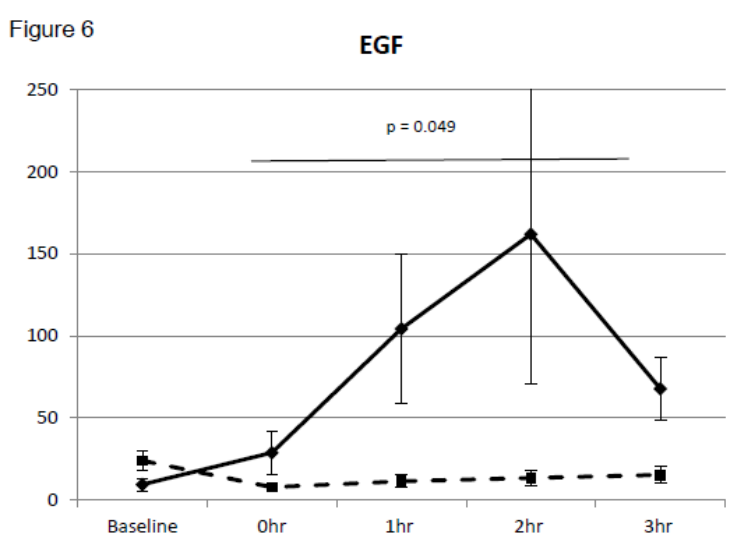

IL-10

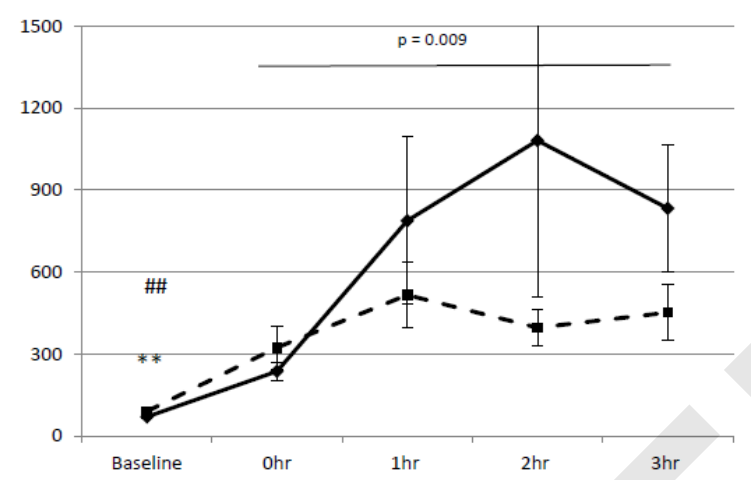

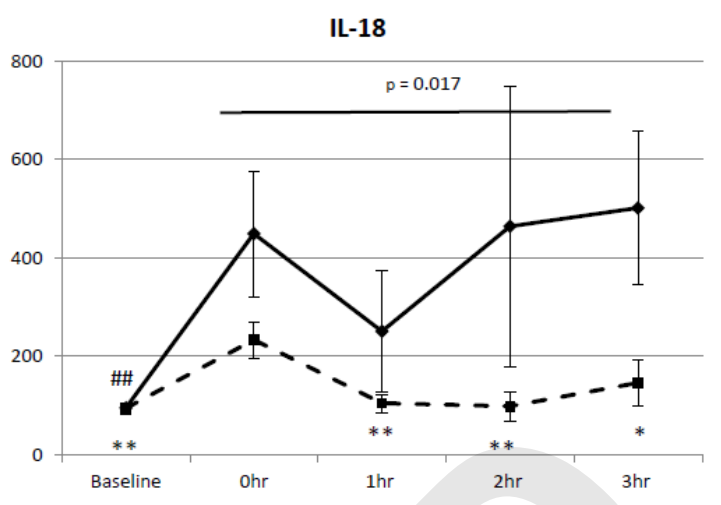

IFN $\gamma$

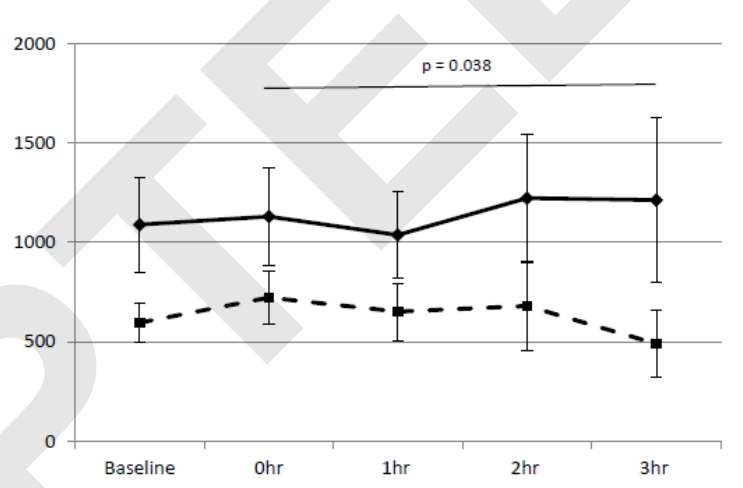

